\title{
The Non-Canonical Effect of $N$-Acetyl-D-Glucosamine Kinase on the Formation of Neuronal Dendrites
}

\author{
HyunSook Lee ${ }^{1}$, Sun-Jung Cho ${ }^{1,3}$, and II Soo Moon ${ }^{1,2, *}$
}

\begin{abstract}
N-acetylglucosamine kinase (GIcNAc kinase or NAGK; EC 2.7.1.59) is a $\mathrm{N}$-acetylhexosamine kinase that belong to the sugar kinase/heat shock protein 70/actin superfamily. In this study, we investigated both the expression and function of NAGK in neurons. Immunohistochemistry of rat brain sections showed that NAGK was expressed at high levels in neurons but at low levels in astrocytes. Immunocytochemistry of rat hippocampal dissociate cultures confirmed these findings and showed that NAGK was also expressed at low levels in oligodendrocytes. Furthermore, several NAGK clusters were observed in the nucleoplasm of both neuron and glia. The overexpression of EGFP- or RFP (DsRed2)-tagged NAGK in rat hippocampal neurons (DIV 5-9) increased the complexity of dendritic architecture by increasing the numbers of primary dendrites and dendritic branches. In contrast, knockdown of NAGK by shRNA resulted in dendrite degeneration, and this was prevented by the co-expression of RFP-tagged NAGK. These results suggest that the upregulation of dendritic complexity is a non-canonical function of NAGK.
\end{abstract}

\section{INTRODUCTION}

$\mathrm{N}$-acetylglucosamine kinase (GlcNAc kinase or NAGK; EC 2.7.1.59) phosphorylates GlcNAc to produce GlcNAc-6-phosphate (GlcNAc-6-P), which is converted to GlcNAc-1-P by GlcNAc-6-P mutase, and GlcNAc-1-P is then utilized to synthesize uridine diphosphate (UDP)-GIcNAc. In mammalian cells, the activated sugar nucleotide UDP-GIcNAc is used to synthesize various oligosaccharide chains, such as $\mathrm{N}-/ \mathrm{O}$-glycans and glycolipids (Hakomori, 2000; Schachter, 2000; Van den Steen et al., 1998), glycosaminoglycans (Esko and Lindahl, 2001), and

\footnotetext{
${ }^{1}$ Department of Anatomy, ${ }^{2}$ Dongguk Medical Institute, Dongguk University College of Medicine, Gyeongju 780-714, Korea, ${ }^{3}$ Present address: Division of Brain Diseases, Center for Biomedical Sciences, Korea National Institute of Health, Cheongwon 363-951, Korea

${ }^{*}$ Correspondence: moonis@ dongguk.ac.kr
}

Received 26 November, 2013; revised 15 January, 2014; accepted 16 January, 2014; published online 12 March, 2014

Keywords: culture, $\mathrm{N}$-acetylglucosamine kinase, neuron, overexpression, shRNA the glycosyl phosphatidylinositol anchor of membrane-bound glycoproteins (Watanabe et al., 2000).

Rat and human NAGKs form homodimers of 37- and 39-kDa subunits, respectively, in solution (Hinderlich et al., 1998). Furthermore, human and mouse genes for NAGK have been cloned (Hinderlich et al., 2000), and a sequence alignment study showed that NAGK is a $\mathrm{N}$-acetylhexosamine kinase (Berger et al., 2002), and thus, a member of the sugar kinase/heat shock protein 70/actin superfamily (Hurley, 1996). This superfamily is defined by a fold consisting of two domains with the $\beta \beta \beta \alpha \beta \alpha \beta \alpha$ Members of this superfamily catalyze ATP phosphoryl transfer or hydrolysis, which is coupled to a large conformational change that induces the two domains to close (Hurley, 1996). Efforts to produce protein crystals of NAGK complexed with the two authentic substrates, GlcNAc and ATP, were unsuccessful (Weihofen et al., 2006). However, two crystal structures of homodimeric human NAGK were described; one complexed with GlcNAc and the other with ADP and glucose. These structures revealed that the secondary structure of human NAGK is comprised of $11 \beta$-strands and ten $\alpha$-helices. Furthermore, the $\mathrm{N}$-terminal small and $\mathrm{C}$-terminal large domains were found to form a V-shaped structure, which acts as an active center for binding GlcNAc and ATP. When complexed with ADP and glucose, NAGK adopts an open configuration, whereas when complexed with GIcNAc, NAGK forms a closed configuration, by rotating the small domain by $26^{\circ}$ relative to the large domain. Using information gleaned from the two crystal structures, Weihofen et al. (2006) established a model structure for NAGK complexed with GIcNAc and ATP/ $\mathrm{Mg}^{2+}$ by superimposing ADP in the ADP/glucose-bound open complex with NAGK in the GlcNAc-bound closed complex. Interestingly, a recent saturation transfer difference NMR study indicated that GlcNAc kinase phosphorylates $\beta$-GlcNAc in cells (Blume, 2008).

NAGK was first characterized as early as in 1970 (Datta, 1970), and since, its characteristics and molecular structure have been studied in detail. However, few studies have addressed the expression or functions of NAGK in mammalian tissues. In one such study, Northern and Western blot analyses showed that NAGK mRNA and protein are expressed throughout various cell lines and tissues (Hinderlich et al., 2000). In addition, immunocytochemistry (ICC) of interphase NIH-3T3 cells revealed NAGK is a cytoplasmic enzyme that is located predominantly in the perinuclear area and at the cell periphery 
(Hinderlich et al., 2000). However, no information is available on the expression or function of NAGK in the nervous system. Accordingly, in the present study, we investigated the expression and function of NAGK in neurons.

\section{MATERIALS AND METHODS}

\section{Antibodies}

The following antibodies were used at the indicated dilutions: MAb microtubule-associated protein 2 (MAP2; 1:250; Sigma); MAb green fluorescent protein [GFP; 1:1000, Chemicon International Inc. (now Millipore, USA)]; MAb neurofilament 200 (NF200) (phosphorylated NF200-specific clone NE14, 1:300; Sigma); rabbit polyclonal glial fibrillary acidic protein (GFAP) (1:300; Sigma); rabbit polyclonal red fluorescent protein (RFP; 1:1000, Chemicon); affinity-pure rabbit polyclonal 2', 3'-cyclic nucleotide 3'-phosphodiesterase (CNPase, 1:500; Cho et al., 2003); Chicken polyclonal NAGK (NAGK) (1:1000 for ICC, 1:300 for IHC; GenWay Biotech, Inc., USA; now GW22347, Sigma).

\section{Substrate competition and immunoblot analysis}

Rat forebrain homogenates $(70 \mu \mathrm{g})$ were separated by electrophoresis in sodium dodecyl sulphate polyacrylamide gel (10\%), proteins were transferred to nitrocellulose (NC) membranes, and membranes were incubated with preblocking solution $[5 \%$ (w/v) skim milk in Tris-buffered saline Tween-20 (TBST; $25 \mathrm{mM}$ Tris, $140 \mathrm{mM} \mathrm{NaCl}, 3 \mathrm{mM} \mathrm{KCl}, 0.05 \%$ Tween-20, $\mathrm{pH}$ 8.0)] for $1.5 \mathrm{~h}$. Separately, NAGK antibody $(1.0 \mu \mathrm{g})$ was mixed with various amounts of purified NAGK in a small volume $(100 \mu \mathrm{l})$ of TBST, and incubated at RT for $1 \mathrm{hr}$ to form antigen-antibody complexes. These mixtures were then added to $3 \mathrm{ml}$ of preblocking solution, and used to blot NC membranes in sealing bags. After incubation overnight at $4^{\circ} \mathrm{C}$, blots were rinsed in TBST $(4 \times 10 \mathrm{~min})$. HRP-conjugated chicken secondary antibody (Perox-AffiniPure Dnk Anti-Chicken IgY (lgG) $(\mathrm{H}+\mathrm{L})$; Jackson ImmunoResearch, USA) was then added and incubated at RT for $1.5 \mathrm{~h}$. The antigen-antibody complex was visualized using the PowerOpti-ECL Western blotting detection kit (Animal Genetics, Inc., Korea).

\section{Plasmids}

\section{Construction of short hairpin (sh)RNA plasmids}

The shRNA and mismatch template oligonucleotide sets were designed as follows; shRNA; Forward: 5'-TTTGCATAAGCC TACAGTTGGAGCCTTTGATATCCGAGGCTCCAACTGTAG GCTTATTTTTTT-3'/Reverse: 5'-CTAGAAAAAAATAAGCCTA CAGTTGGAGCCTCGGATATCAAAGGCTCCAACTGTAGGC TTATG-3'. Negative control (mismatch); Forward: 5'-TTTG CATGAGCCTGCAGTAGGATCCTTTGATATCCGAGGATCC TACTGCAGGCTCATTTTTTT-3'/Reverse: 5'-CTAGAAAAAAA TGAGCCTGCAGTAGGATCCTCGGATATCAAAGGATCCTA CTGCAGGCTCATG-3' (mismatches are underlined). Oligonucleotide sets were annealed and inserted into the $\mathrm{Bbsl} / \mathrm{Xbal}$ site of mU6pro vector (Yu et al., 2002, a gift from Dr. David L. Turner at the University of Michigan, Ann Arbor).

Primary culture, fixation, and transfection of rat hippocampal neurons

Hippocampi from embryonic day 18 (E18) or E19 SpragueDawley rat pups were dissected, dissociated by trypsin treatment and mechanical trituration, and plated onto 12-mm diameter polylysine/laminin-coated glass coverslips at a density of $\sim 150$ neurons $/ \mathrm{mm}^{2}$, as previously described (Brewer et al., 1993) Cells were initially plated in Neurobasal medium supplemented with B27 (Invitrogen, USA), $25 \mu \mathrm{M}$ glutamate, and $500 \mu \mathrm{M}$ glutamine, and fed 5 days after plating and weekly thereafter with the same media (without added glutamate) containing $1 / 3$ (v/v) Neurobasal medium preconditioned by incubation for $24 \mathrm{~h}$ in astrocyte culture, as previously described (Cho et al., 2011; Goslin et al., 1998). After 21 days in vitro (DIV), coverslips were rinsed briefly in Dulbecco's phosphate-buffered saline (Invitrogen) and fixed using a sequential paraformaldehyde/methanol fixation procedure [incubation in $4 \%$ paraformaldehyde in PBS (20 mM sodium phosphate buffer, $\mathrm{pH} 7.4,0.9 \% \mathrm{NaCl}$ ) at room temperature (RT) for $10 \mathrm{~min}$, followed by incubation in methanol at $-20^{\circ} \mathrm{C}$ for $20 \mathrm{~min}$ ] (Moon et al., 2007). Transfection was carried out as described by Jiang et al. (2004), using the ClonTech CalPhos ${ }^{\mathrm{TM}}$ Mammalian Transfection Kit (BD Bioscience, USA).

\section{Immunocytochemistry (ICC)}

Cells were fixed using the sequential paraformaldehyde/methanol fixation procedure at RT for $10 \mathrm{~min}$ and then incubated in methanol at $-20^{\circ} \mathrm{C}$ for 20 min (Moon et al., 2007). ICC was performed with the indicated primary and secondary antibodies [Alexa Fluor 488-conjugated goat anti-mouse, Alexa Fluor 568conjugated goat anti-rabbit, and Alexa Fluor 647-conjugated goat anti-chicken IgG (each diluted 1:1,000 in blocking buffer; Invitrogen)], as previously described (Moon et al., 2007).

\section{Immunohistochemistry (IHC)}

Male adult (8-week-old; 250-300 g) Sprague-Dawley rats were anesthetized under isoflurane/ $\mathrm{N}_{2} \mathrm{O}$ and brain tissues were fixed with a transcardiac infusion of $4 \%$ paraformaldehyde in PBS $(\mathrm{pH}$ 7.4). The perfusion-fixed brains were removed, post-fixed overnight in the same fixative at RT, and paraffin-embedded. Serial sagittal $5 \mu \mathrm{m}$ sections were then prepared and mounted on slides. After deparaffinization, sections were incubated in blocking solution $(1.0 \%$ BSA, $0.2 \%$ gelatin, $0.05 \%$ saponin in PBS) $3 \times 30 \mathrm{~min}$ at RT, and then incubated with primary antibodies [chick polyclonal NAGK (1:300), MAb GFAP, and NF200 (both 1:300)] diluted in 0.1\% BSA, 0.3\% Triton X-100 in PBS overnight at $4^{\circ} \mathrm{C}$. The following day, sections were rinsed $(3 \times 10 \mathrm{~min})$ in $0.1 \% \mathrm{BSA}, 0.2 \%$ gelatin, $0.05 \%$ saponin in PBS, and then incubated with secondary antibodies [Alexa Fluor 488-conjugated goat anti-mouse, Alexa Fluor 568-conjugated goat anti-rabbit, and Alexa Fluor 647-conjugated goat antichicken $\operatorname{lgG}$ (each 1:1,000)] diluted in $0.1 \% \mathrm{BSA}, 0.3 \%$ Triton $\mathrm{X}-100$ in PBS for $1 \mathrm{~h}$ at RT. After rinsing in $0.1 \% \mathrm{BSA}, 0.2 \%$ gelatin, $0.05 \%$ saponin in PBS $(3 \times 10 \mathrm{~min})$, sections were washed in PBS ( $3 \times 10 \mathrm{~min})$ and mounted using prewarmed fade-retarding mounting solution [100 mg/ml DABCO (1,4-diazavicyclo [2.2.2] octane; Sigma) in $90 \%$ glycerol and $10 \%$ PBS (pH 7.4)].

\section{Light and laser-scanning confocal microscopy}

A Leica Research Microscope DM IRE2 equipped with I3 S, N2.1 S, and Y5 filter systems (Leica Microsystems AG, Germany) was used for epifluorescence microscopy. Images (1388 $\times 1039$ pixels) were acquired using a high-resolution CoolSNAPTM CCD camera (Photometrics Inc., USA) under the control of a computer running Leica FW4000 software. Confocal images (1024 $\times 1024$ pixels) were acquired using a Leica TCS SP2 confocal system with laser lines at 488,543 , and $633 \mathrm{~nm}$. Digital images were processed using Adobe Systems Photoshop 7.0. 


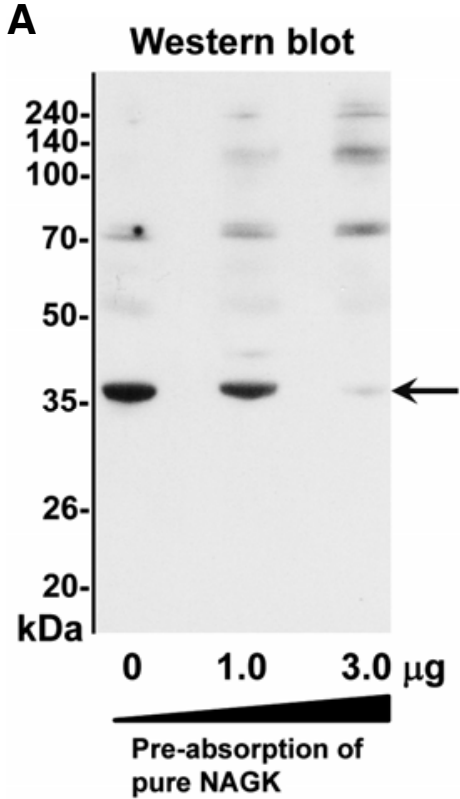

B

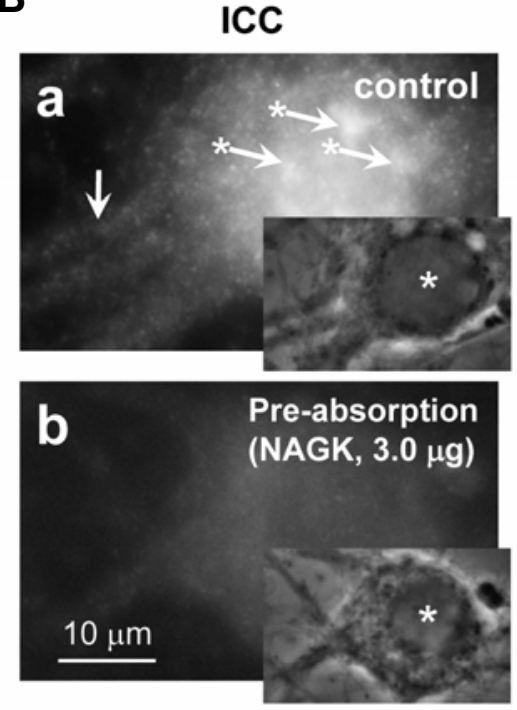

anti-NAGK
Fig. 1. Antibody specificity. (A) Western blotting. Rat forebrain homogenates $(70 \mu \mathrm{g})$ were electrophoresed in $10 \%$ SDS-polyacryl-amide gels, transferred to NC membranes, which were immunoblotted with anti-NAGK antibody previously exposed to different amounts of purified NAGK $(0$, $1.0,3.0 \mu \mathrm{g})$. Note that the signal intensity of NAGK at $37 \mathrm{kD}$ (arrow) decreased dose-dependently. Molecular size is indicated on the left (kDa). (B) Immunocytochemistry (ICC). Cultured rat hippocampal neurons (DIV 21) were immunostained with anti-NAGK antibody directly (control; a) or anti-NAGK antibody pre-exposed to purified NAGK (3.0 $\mu \mathrm{g}$; b). Nuclear (arrow with an asterisk) and dendrite (arrow) NAGK clusters are indicated (a). These NAGK clusters disappeared or markedly weakened when the anti-NAGK antibody was pretreated with purified NAGK (b). Insets show phase contrast images, in which nuclei are marked with asterisks.

\section{Analysis}

To determine the degrees of arborization of dendritic tree, we counted numbers of primary dendrites and their branches as described by Sholl (1953). The dendritic intersection is defined as the point where primary dendrites or their branch intersects a given concentric circle. The numbers of dendritic branches intersecting two successive concentric circles were counted. Transfected neurons (a minimum of 15 cells) were selected for analysis. The Mann-Whitney $U$-test was used to determine statistical significances, and $p$ values of $<0.05$ and $<0.01$ were considered to be significant or highly significant, respectively.

\section{RESULTS}

Verification of antibody specificity by antigen blocking We used a commercial antibody against NAGK, but because of a lack of published information, we first tested the specificity of this antibody. For this purpose we first immune-neutralized the antibody with antigens. Briefly, the antibody $(1.0 \mu \mathrm{g})$ was mixed with increasing amounts of antigens $(0,1.0$, or $3.0 \mu \mathrm{g}$ of pure NAGK) in a small volume (100 $\mu$ l) to block the antigen-binding site. These mixtures were then used for immunoblotting rat forebrain homogenates on NC membranes. As shown by immunoblot images, the antibody specifically recognized a band at $37 \mathrm{kDa}$ (Fig. 1A, $0 \mu \mathrm{g}$ ). This $37 \mathrm{kDa}$ band was gradually weakened by pre-treating membranes with increasing amounts of pure NAGK $(1.0$ and $3.0 \mu \mathrm{g})$, indicating that the antibody specifically binds NAGK. We further tested antibody specificity by immunocytochemistry (ICC). Typically, ICC images of hippocampal neurons reveal small punctae in the somatodendritic domain (arrowed in Fig. 1B-a) and several large nuclear clusters (asterisked arrows in Fig. 1B-a). When the antibody was neutralized by preincubation with its antigen (pure NAGK, 3.0 $\mu \mathrm{g}$ ), the intensity of the NAGK-immunoreactive (IR) signal in the somatodendritic domain weakened significantly and the nuclear NAGK clusters were not detected (Fig. 1B-b). These data demonstrate that the antibody used bound specifically to NAGK.

\section{NAGK was highly expressed in neurons}

\section{Immunohistochemistry (IHC)}

Having confirmed the specificity of the NAGK antibody, we investigated the expression of NAGK in brain cells by IHC. Rat brain slices were triple-labeled with antibodies against NAGK, NF200, and GFAP (Figs. 2 and 3A). When IR signals of NAGK and NF200 (a neuronal marker) were compared, strong NAGKIR signals were found to be well co-localized with NF200-positive $(+)$ cells, that is, with pyramidal cells in the cerebral cortex (Cb) (Fig. 2A, arrowheads), Purkinje cells and granule cells in the cerebellum (Cbl) (Fig. 2B, arrowheads and arrows, respectively), the soma and dendrites of pyramidal cells in the hippocampal CA1 region (Fig. 2C, asterisk and arrowhead, respectively), granule cells in the dentate gyrus (DG), and polymorphic cells in the dentate hilus (DH) (Fig. 2D, asterisk and arrowhead, respectively). In the CA1 region,a strong association between NAGK-IR signals and the stratum radiatum (SR) and the stratum pyramidale (SP) (Fig. 2C, arrowhead and asterisk, respectively) was evident. In contrast, NAGK-IR signals were weak in astrocytes,for example, in the hippocampal CA1 region, strong NAGK-IR signals (Fig. 3A, arrows) were not co-localized with GFAP(+) cells (Fig. 3A, asterisks). These results indicate that NAGK is highly expressed in neurons, but relatively weakly expressed in astrocytes.

\section{Immunocytochemistry (ICC)}

To confirm the strong expression of NAGK in neurons, we conducted ICC on rat hippocampal dissociated cultures. NAGK-IR signals were completely separated from GFAP (an astrocyte marker, Fig. 3B) and CNPase (an oligodendrocyte marker, Fig. 3C) signals. Instead, strong NAGK-IR cells were positive for the MAP2 (a neuronal marker, Fig. 3D). The intensities of NAGK-IR signals in the cytoplasms of GFAP(+) and CNPase(+) cells were very weak (Figs. 3B and $3 C$ ). Interestingly, several NAGKIR clusters were present in the nuclei of neuronal (arrowhead in Fig. 5C, NAGK) and non-neuronal cells (astrocytes, arrowhead 


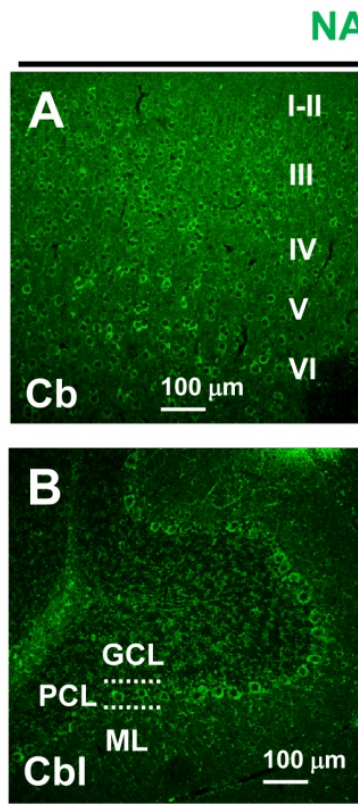

NAGK
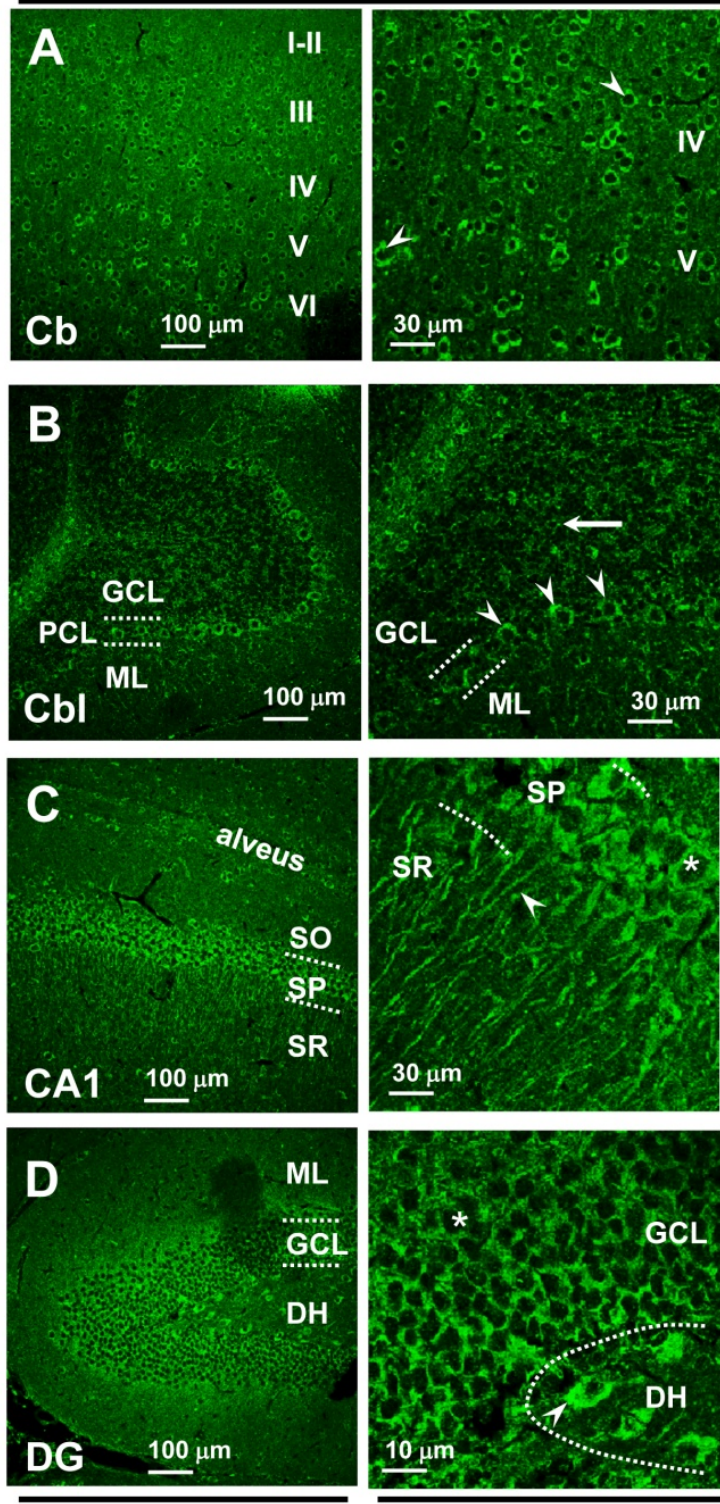

20X objective
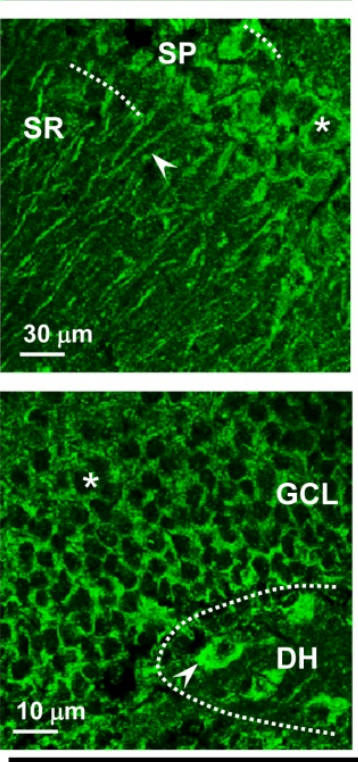

40X objective
Fig. 2. Immunohistochemical analysis of NAGK expression in rat brain sections. Rat brain sections were labeled with antibodies against NAGK and NF200. Confocal microscope images of NAGK and NF200 labeling were obtained using 20X (left column) or $40 \mathrm{X}$ objective lenses (right two columns). (A) NAGK distribution in the rat cerebrum (Cb). Arrowheads, pyramidal neurons. Laminar layers are indicated by Roman numerals. (B) NAGK distribution in the rat cerebellum (Cbl). Arrowheads are Purkinje cells and the arrow indicates a granule cell. GCL, granule cell layer; PCL, Purkinje cell layer; ML, molecular layer. (C) NAGK distribution in hippocampal CA1 region. The asterisk indicates a soma and arrowheads the dendrites of pyramidal neurons. SO, stratum oriens; $\mathrm{SP}$, stratum pyramidale; SR, stratum radiatum. (D) NAGK distribution in the rat dentate gyrus (DG). The asterisk indicates a granule cell and the arrowhead a polymorphic cell. ML, molecular layer; GCL, granular cell layer; $\mathrm{DH}$, dentate hilus.
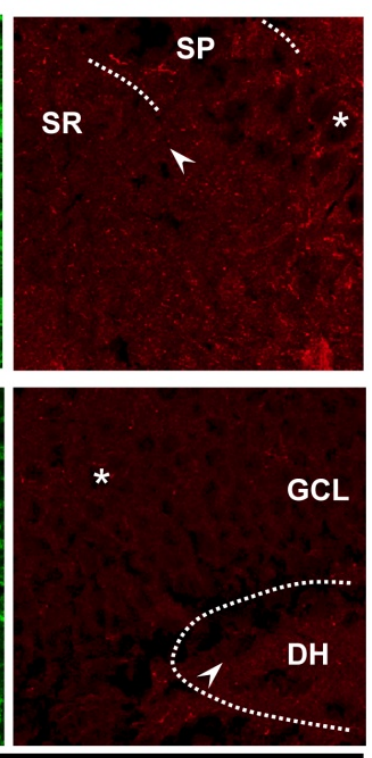

GCL in Fig. 3B; oligodendrocytes, arrowhead in Fig. 3C, NAGK, inset). Furthermore, these nuclear NAGK-IR clusters were also observed in cells in hippocampal dissociated cultures and in other cell types in culture, including HEK293T cells and several cancer cell lines (data not shown). Together, these results confirm that NAGK is highly expressed in neurons.

\section{NAGK overexpression upregulated the number of dendrites}

The strong expression of NAGK observed in neuronal dendrites prompted us to investigate its possible functions in neurons. First, we investigated NAGK gain-of-function. To this end, we introduced by transfection EGFP- or RFP (DsRed)-tagged NAGK genes into rat hippocampal neurons (DIV 5-9) in culture.
Surprisingly, live cell images of the transfected neurons at 16$48 \mathrm{~h}$ post-transfection exhibited increased numbers of dendrites on EGFP- and DsRed-tagged NAGK overexpressing neurons (Fig. 4A). Furthermore, the neurons overexpressing NAGK of either tag, exhibited more complex cytoarchitectures than control neurons. The number of primary dendrites increased by $32 \%$ (from 8.4 to 11.1) in DsRed2-transfected neurons and by $28 \%$ (from 9.6 to 12.3) in EGFP-NAGK transfected neurons (Fig. 4Ba), and these increases were highly significant $(p<0.01)$. The number of dendritic intersections crossing $60 \mu \mathrm{m}$ circles from the centroid of soma also increased by $115 \%$ (from 11.2 to 24.1 ) in DsRed2-transfected neurons and by $55 \%$ (from 15.5 to 24.1 ) in EGFP-NAGK transfected neurons (Fig. 4B-b), and these increases were also highly significant $(p<0.01)$. 

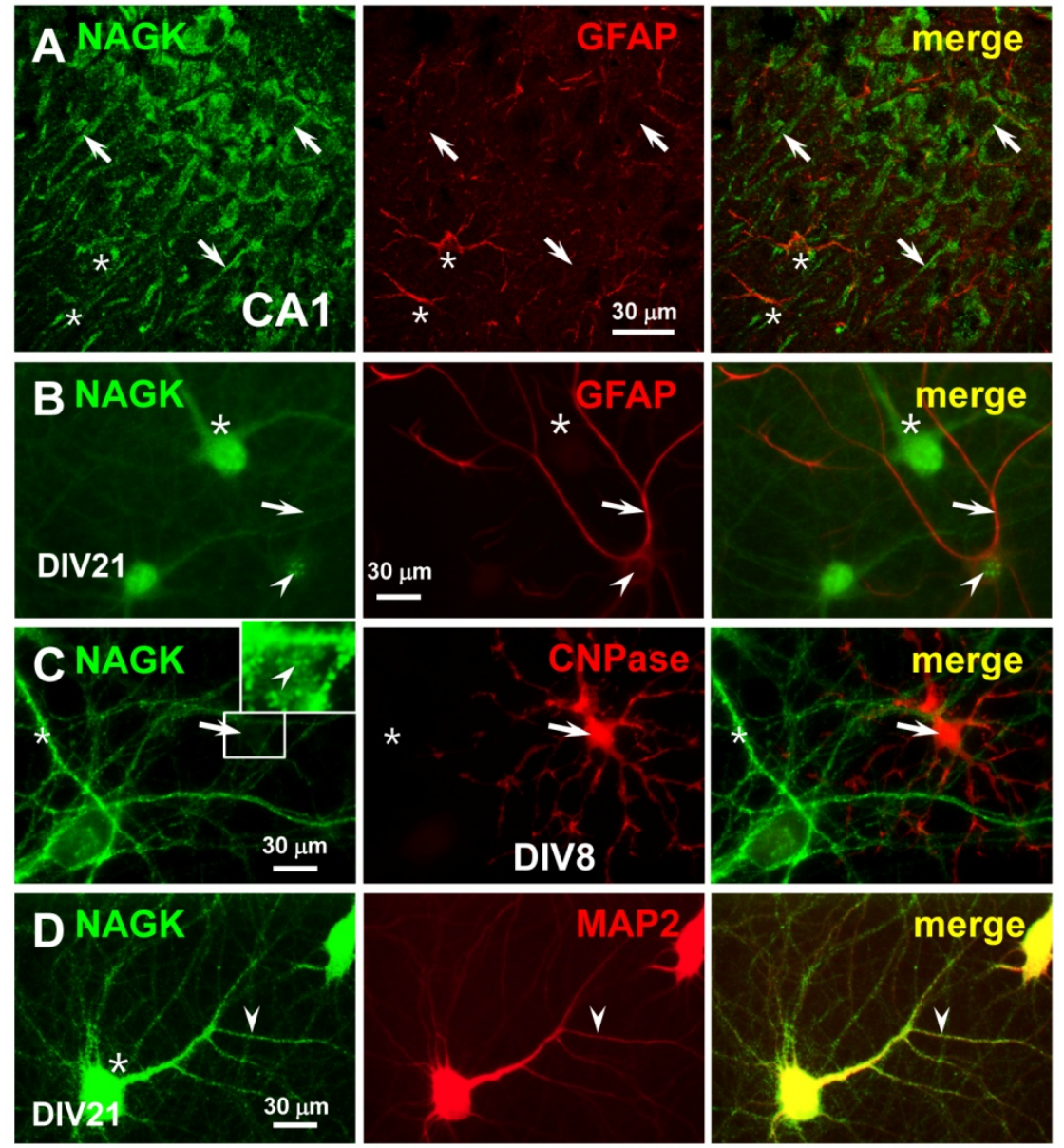

Fig. 3. Elevated expression of NAGK in neurons. (A) Immunohistochemistry (IHC). The confocal images of NAGK and GFAP labeling in the rat CA1 region (the NAGK image is a crop of the image in Fig. $2 \mathrm{C}$ ) showing complete separation of the two IR signals. Arrow, pyramidal neurons; Asterisk, GFAP-positive astrocyte. (B-D) Immunocytochemistry (ICC). Dissociated rat hippocampal cultures (DIV21) were double-labeled, as indicated. Neuronal soma and dendrites are indicated by asterisks. Astrocytes and oligodendrocytes were identified by immunostaining with GFAP (B, arrow) and CNPase (C, arrow), respectively. NAGK-IR was completely separated from GFAP-IR and from CNPaseIR. In contrast, NAGK-IR signals co-localized well with MAP2 (D, arrowhead), a neuronal marker. NAGK clusters in the nucleus of an astrocyte (GFAP) and oligodendrocyte (CNPase) are indicated by arrowheads in (B) and (C) (and highly contrasted images in inset, respectively). Scale bars, $30 \mu \mathrm{m}$.
A

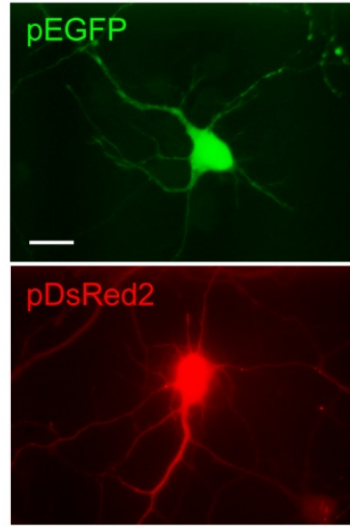

B

a

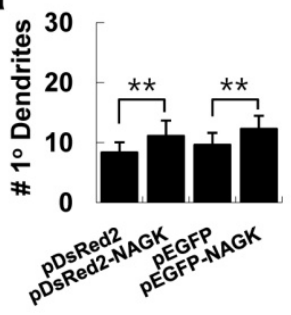

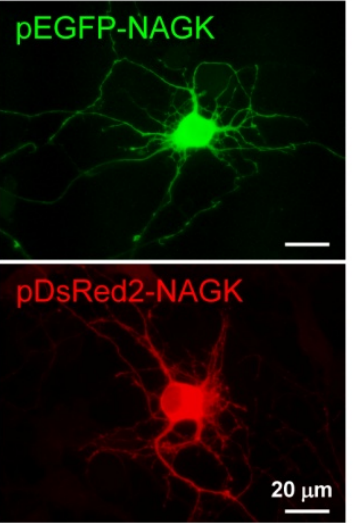

b

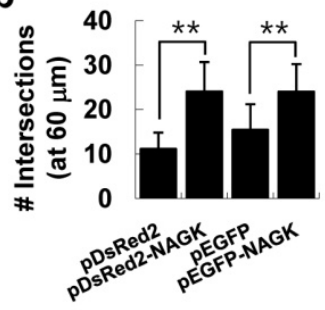

Fig. 4. Overexpression of NAGK increased dendritic complexity. (A) Epifluorescent live cell images showing neurons transfected with the indicated plasmids. (B) Statistics. Numbers of primary dendrites (a) and of dendritic branches crossing circles at $60 \mu \mathrm{m}$ distance from the soma centroids (b) were counted. ${ }^{* *} p<0.01$. 
A
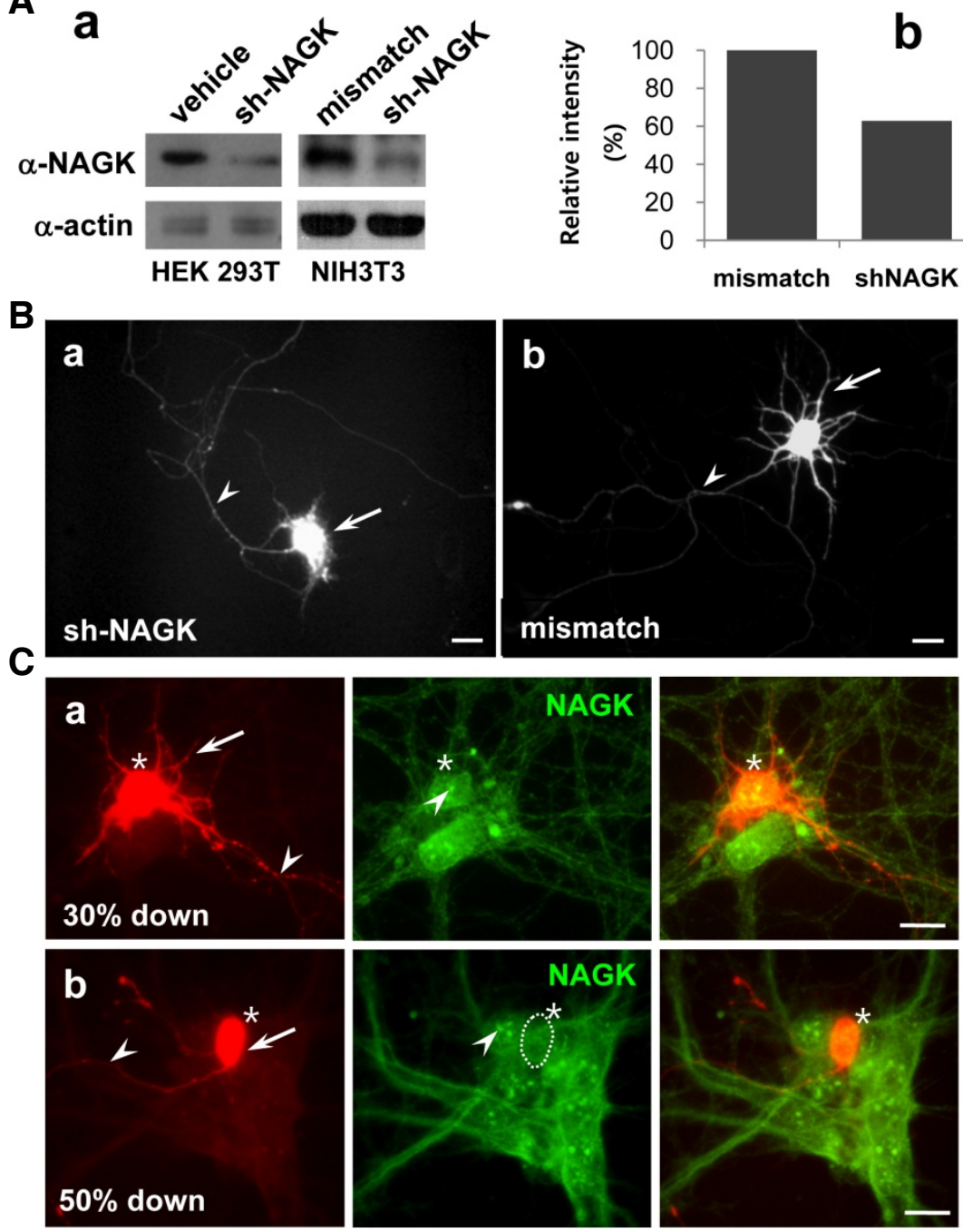

D

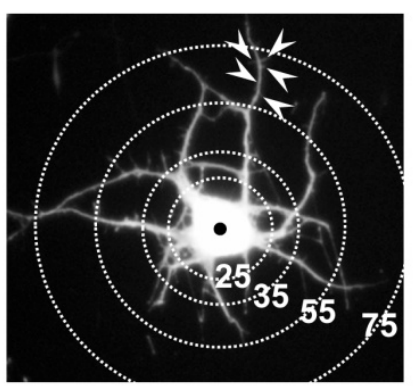

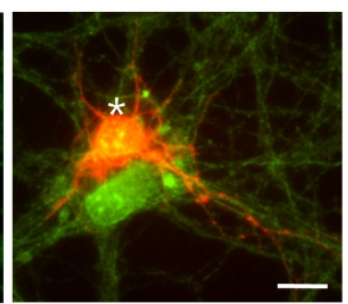

b

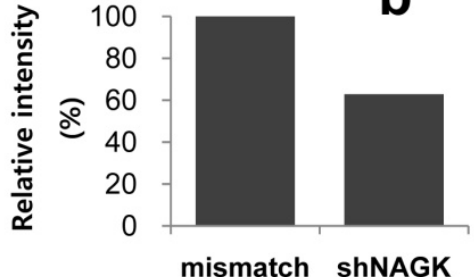

Fig. 5. Knockdown of NAGK mRNA induced dendrite degeneration. (A) Immunoblots. HEK293T or NIH3T3 cells were co-transfected with DsRed2 vehicle or DsRed2 + the indicated vectors, and immunoblotted with $\alpha$ NAGK. Membranes were stripped of the antibody and reblotted with $\alpha$-actin (a). The immunoblot signal intensities of NIH3T3 were measured by densitometry and normalized versus mismatch vehicle. Data are averages of duplicate experiments (b). (B-D) shRNA experiments. An epifluorescence image of a typical neuron co-transfected with DsRed2 + NAGK shRNA vectors (sh-NAGK) showing dramatic dendrite degeneration (arrow), but an intact axons (arrowhead) (Ba). In contrast, the dendrite (arrow) and axon (arrowhead) of a control neuron transfected with mismatch vector were intact (B-b). Cultured hippocampal neurons were cotransfected with DsRed2 + sh-NAGK vectors, and double-stained with $\alpha$-RFP and $\alpha$ NAGK antibodies. Images of neurons (red) showing $\sim 30 \% \quad(\mathrm{C}-\mathrm{a}$, arrow) and $\sim 50 \%$ knockdown (C-b, asterisk) of NAGK as compared with neighboring neurons (green) (obtained by densitometry). Note that dendrites (arrow) were dramatically shorter after 30\% knockdown (C-a) and were almost completely degenerated after 50\% knockdown (C-b, asterisk), whereas axons (arrowheads) were relatively unaffected. Numbers of dendritic branches at distances of 25-35, 35-55, $55-75$, > $75 \mu \mathrm{m}$ from soma centroids of DsRed2 control and shRNA expressing neurons (sh-NAGK) (D). ${ }^{* \star} p<0.01$. Scale bar, $20 \mu \mathrm{m}$.
These results indicate that when analyzed at $60 \mu \mathrm{m}$ from the center of soma, branching frequencies (i.e. numbers of intersections divided by numbers of primary dendrites) also increased from 1.3 (control; 11.2 divided by 8.4 ) to 2.2 (24.1 divided by 11.1) in DsRed2-transfected neurons and from 1.6 (control; 15.5 divided by 9.6 ) to 2.0 (24.1 divided by 12.3 ) in EGFP-NAGK transfected neurons. Taken together, these results indicate that NAGK increases the complexity of dendritic architecture by increasing the numbers of primary dendrites and dendritic branches.
Knockdown of NAGK resulted in dendrite degeneration Next, we investigated loss-of-function of NAGK in neurons. One set of forward and reverse primers was designed to produce NAGK shRNA (sh-NAGK); the control set used contained mismatches. When human HEK293T or mouse NIH3T3 cells were transfected with sh-NAGK vector (transfection efficiency, > 80\%), the expression of NAGK was significantly reduced by Western blotting, whereas reductions were negligible for vehicle and for mismatch vectors (Fig. 5A-a). Western blotting showed that the expression of NAGK was reduced to $\sim 60 \%$ of the mismatch 
A
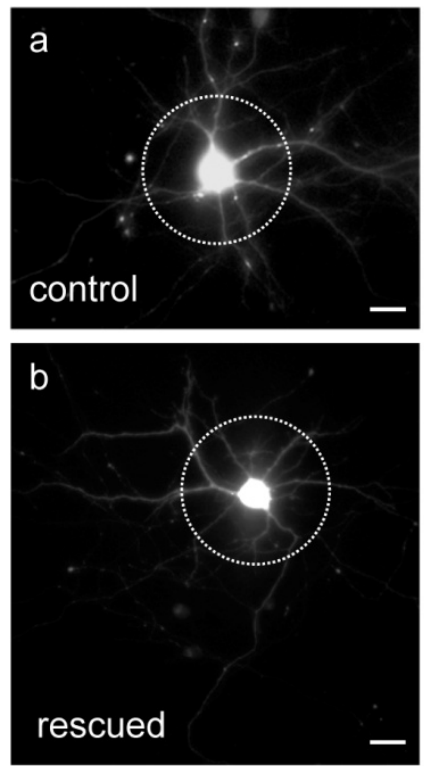

B

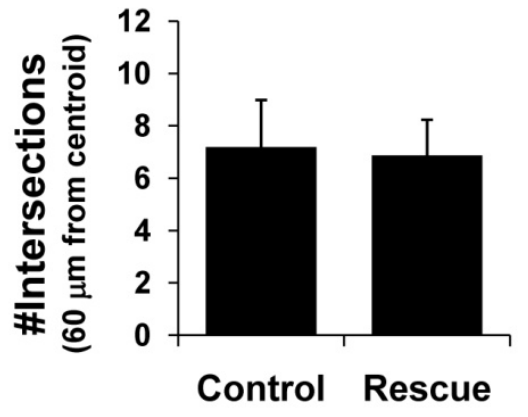

Fig. 6. Rescue experiments. (A) Epifluorescence images of a typical live neuron transfected with control (DsRed2) (a) or co-transfected with sh-NAGK + DsRed2-NAGK (rescued, b) vectors. (B) Numbers of dendrites crossing the circle at $60 \mu \mathrm{m}$ from the centroid of soma were counted. Scale bar, $20 \mu \mathrm{m}$. control by sh-NAGK in NIH-3T3 cells (Fig. 5A-b). To investigate the knockdown effect of NAGK, these vectors were introduced, together with DsRed2 plasmid for identification purposes, by co-transfection into rat hippocampal neurons in culture (DIV 59). Surprisingly, neurons transfected with sh-NAGK exhibited dramatic dendrite degeneration (Fig. 5B-a, arrow), though axons were less affected (Fig. 5B-a, arrowhead). On the other hand, the control mismatch vector had no noticeable effect (Fig. 5B-b). Furthermore, the observed dendritic degeneration was proportional to reductions in NAGK protein levels. For example, when the expression level of NAGK was reduced by $\sim 30 \%$ (as determined by densitometry of ICC signals), as compared with neighboring untransfected neurons, neurons (Fig. 5C-a, asterisk) still retained some short dendrites (arrow). Also, when a transfected neuron exhibited a reduction in NAGK level by $50 \%$ (Fig. 5C-b, asterisk), it showed almost complete dendrite degeneration (arrow), although once again axons were apparently less affected (Fig. 5C, arrowheads). When dendritic branches were counted at distances of 25-35, 35-55, 55-75, > $75 \mu \mathrm{m}$ ) from soma centroids, sh-NAGK RNA-transfected neurons showed significantly $(p<0.01)$ fewer dendrites at all distances, except at 25-35 $\mu \mathrm{m}$ (Fig. 5D). These results indicate that dendrites degenerate progressively from the distal to proximal in the presence of NAGK deficiency.

Overexpression of NAGK prevented the effects of shRNA To determine whether dendritic degeneration was specifically caused by NAGK knockdown, we investigated whether the overexpression of NAGK in neurons could abrogate the effect of shRNA. To this end, we co-transfected cultured rat hippocampal neurons with sh-NAGK and DsRed2-NAGK vectors; examples of transfected neurons are shown in Fig. 6A. The morphology of the rescued cells (Fig. 6A-b) was similar to that of DsRed2-transfected control neurons (Fig. 6A-a). Statistical analysis also showed no significant difference between DsRed2 control and sh-NAGK plus DsRed2-NAGK groups (7.8 and 6.8 , respectively; Fig. 6B-b) with respect to the mean number of dendritic intersections crossing a circle at $60 \mu \mathrm{m}$ from the centroid of soma. This result confirmed that the degeneration effect was specifically caused by NAGK knockdown.

\section{DISCUSSION}

This study shows that NAGK is highly expressed in brain neurons, but poorly expressed in neuroglia, such as, astrocytes and oligodendrocytes, and that NAGK plays a critical role in the development of neuronal dendrites. Many proteins have multiple functions, and the canonical function of NAGK is the phosphorylation of GlcNAc to produce GlcNAc 6-phosphate. However, the present study provides evidence for a non-canonical function of NAGK in the development of neuronal dendrites.

Few studies have been conducted on the expression of NAGK in mammalian cells. However, available data indicate that NAGK is ubiquitously expressed in mammalian cells. In one study, NAGK mRNA was detected by Northern blot in various human cancer cell lines, including promyelotic leukemia HL-60, HeLa S3, chronic myelogenous leukemia K562, lymphoblastic leukemia MOLT-4, Burkitt's lymphoma Raji, colorectal adenocarcinoma SW 480, lung carcinoma A549, and melanoma G361 (Hinderlich et al., 2000). NAGK protein was also detected by Western blot in diverse murine cell lines, such as, B cells at several maturation stages (FL5.12, WEHI, A.20), a T cell line (EL4), normal (NIH3T3), or transformed fibroblasts (BALB3T3-SV40), an endothelial cell line (ST2), and in the monkey COS-1 cell line (Ligos et al., 2002). We also found by immunoblotting that NAGK was expressed in all tissues tested, which included brain, heart, kidney, liver, lung, skeletal muscle, spleen, and testes (our unpublished data). This ubiquitous expression of NAGK implies that the enzyme plays a housekeeping function, which probably involves the phosphorylation of NAG in carbohydrate metabolism. A nuclear house-keeping function is also likely because we observed ubiquitous nuclear expression in the present study. More specifically, our ICC study showed NAGK clusters in the nucleoplasm of neuronal and non-neuronal cells, and the presence of these nuclear NAGK clusters in all cell types examined suggests NAGK also 
has a house-keeping nuclear function. However, nucleoplasm has many different functional subdomains, and thus, a detailed study on the subnuclear localization of NAGK is under way in our laboratory.

NAGK expression depends on tissue and cell type. This study shows that NAGK is strongly expressed in neurons as compared with non-neuronal cells. Furthermore, our results show that the commercial antibody against NAGK (now GW22347, Sigma), which we used in this study, is highly specific. Using this specific antibody we found, by IHC of rat brain and rat hippocampal dissociate cultures, that NAGK is highly expressed in brain neurons, which implies that NAGK plays a special role in neurons. In our gain-of-function study, the overexpressions of EGFP- or RFP (DsRed2)-tagged NAGK upregulated dendrite numbers, whereas in the loss-of-function study, knockdown of NAGK by shRNA resulted in dramatic dendrite degeneration. In addition, the overexpression of NAGK resulted in an increase in the numbers (32\% by RFP-NAGK and $28 \%$ by EGFP-NAGK) of primary dendrites (stemming directly from soma), which gave neurons a hairy appearance. Numbers of dendritic branches also increased significantly (115\% by RFPNAGK and $55 \%$ by EGFP-NAGK). These results indicate that NAGK has the ability to generate both primary dendrites and branches. Furthermore, the extent of dendritic degeneration was proportional to the degree of NAGK knockdown. For example, 50\% NAGK knockdown neuronswere almost complete free of dendrites, whereas $30 \%$ NAGK knockdown neurons retained some short dendrites. Moreover, the overexpression of NAGK abrogated the effect of shRNA and prevented dendrite degeneration. These results suggest that the down-regulation of NAGK retards the building and/or maintenance of dendrites, and thus, results in their degeneration.

Our finding that the downregulation of NAGK did not significantly affect axonal morphology is of interest. We will not elaborate on this issue because axons had already grown to a considerable length at the time of transfection (i.e. DIV 5-9). However, it was evident that axons were not markedly shortened. This observation caused us to ask why the downregulation of NAGK caused dendrite and not axon degeneration. Neurons are highly polarized and possess axons and dendrites which differ in terms of morphology and function. In a previous study, a series of time-lapse video microscopic studies on low density cultures of hippocampal neurons revealed a stereotyped sequence of characteristic morphological changes (Dotti et al., 1998; Goslin and Banker, 1990). During the initial several days of culture, axons continue to grow to substantial distances, whereas the processes that become dendrites begin to elongate later and at a significantly lower rate. Furthermore, during the early stage of dendritic development, neurons show dramatic re-arrangements in dendritic arbors, such as, the addition of new branches, complete retraction of branches, and extension or shortening of branches (O'Rourke, 1994). In our shRNA and overexpression experiments, we used DIV 5-9 neurons, and during this period neurons undergo extensive dendritic growth and branching. This suggests that a deficiency or excess of NAGK could accelerate the processes responsible for the retraction or elaboration of dendrites. Our preliminary data suggests that the axonal expression of NAGK decreases dramatically as neurons mature, which suggests that its lesser effect on axons could be due to its differential distribution in subcellular domains. Detailed study on the relation between the subcellular expression of NAGK and developmental stage is in progress in our laboratory.

Our results imply that dendrites, which are morphologically complex and dynamic, are more affected than axonal shafts that have already matured, which suggests NAGK plays a concentration-dependent role in the building and/or maintenance of cell structures, and in the acceleration or retardation of these processes. Mechanistically, we surmise NAGK may function as a member of the metabolic sensor system. NAGK is involved in carbohydrate metabolism that produces UDP-GlcNAc, which is utilized by $O$-GlcNAc transferase (OGT) for $O$-GlcNAcylation. $O$-GlcNAcylation would play the role of a 'rheostat' and delicately tune signal transductions generated in response to cellular nutrient or stress (Hart et al., 2011). Since the overall activity of OGT is regulated linearly by a wide range of intracellular concentrations of UDP-GlcNAc (Kreppel and Hart, 1999), NAGK overexpression could sensitize O-GlcNAcylation and eventually upregulate dendritogenesis. Recent evidence indicates that $O$ GlcNAc could be further modified by phosphorylation (reviewed by Moon et al., 2013). Furthermore, the conversion of O-GlcNAc to $O$-GlcNAc-P by NAGK could increase the effect of $O$ GlcNAcylation. Another possibility is that NAGK plays a structural role as a component of molecular motor. High resolution confocal microscopic images of double-stained neurons revealed that NAGK forms puncta that always localize to or alongside microtubule fibers in dendrites and developing axon and growth cones (manuscript in preparation). Given the fact that NAGK's role is most manifest in dynamic structures undergoing elongation or retraction, it is conceivable that NAGK is a component of a molecular motor complex that transports cargoes along microtubules. Further investigations of this topic are being conducted in our laboratory.

In conclusion, our cell and molecular cell biological studies show that; 1) NAGK is highly expressed in neurons but relatively weakly expressed in glia, 2) the results of our overexpression, shRNA, and rescue experiments indicate that NAGK plays a critical role in the development of dendrites, and 3) NAGK was found to ubiquitously form clusters in nucleoplasm. To the best of our knowledge, this is the first study to address the expression and function of NAGK in the nervous system, and it shows that the promotion of dendritogenesis by NAGK represents a novel non-canonical function.

\section{ACKNOWLEDGMENTS}

We thank Eun-Jung Jung for technical assistance. This research was supported by the Basic Science Research Program of the National Research Foundation (NRF) of Korea funded by the Korean Ministry of Education, Science and Technology (Grant no. NRF-2012R1A1A2006116).

\section{REFERENCES}

Berger, M., Chen, H., Reutter, W., and Hinderlich S. (2002). Structure and function of $N$-acetylglucosamine kinase. Identification of two active site cysteines. Eur. J. Biochem. 269, 4212-4218.

Blume, A., Berger, M., Benie, A.J., Peters, T., and Hinderlich, S. (2008). Characterization of ligand binding to $N$-acetylglucosamine kinase studied by STD NMR. Biochemistry 47, 1313813146.

Brewer, G.J., Torricelli, J.R., Evege, E.K., and Price, P.J. (1993). Optimized survival of hippocampal neurons in B27-supplemented Neurobasal, a new serum-free medium combination. $J$. Neurosci. Res. 35, 567-576.

Cho, S.J., Jung, J.S., Jin, I., and Moon, I.S. (2003). 2', 3'-cyclic nucleotide 3 '-phosphodiesterase is expressed in dissociated rat cerebellar cells and included in the postsynaptic density fraction. Mol. Cells 16, 128-135.

Cho, S.J., Lee, H., Dutta, S., Song, J., Walikonis, R., and Moon, I.S. (2011). Septin 6 regulates the cytoarchitecture of neurons through localization at dendritic branch points and bases of pro- 
trusions. Mol. Cells 32, 89-98.

Datta, A. (1970). Studies on hog spleen $\mathrm{N}$-acetylglucosamine kinase. I. Purification and properties of $\mathrm{N}$-acetylglucosamine kinase. Biochim. Biophys. Acta 220, 51-60.

Dotti, C.G., Sullivan, C.A., and Banker, G.A. (1998). The establishment of polarity by hippocampal neurons in culture. J. Neurosci. 8, 1454-1468.

Esko, J.D., and Lindahl, U. (2001). Molecular diversity of heparan sulfate. J. Clin. Invest. 108, 169-173.

Goslin, K., and Banker, G. (1990). Rapid changes in the distribution of GAP-43 correlate with the expression of neuronal polarity during normal development and under experimental conditions. J. Cell Biol. 110, 1319-1331.

Goslin, K., Assmussen, H., and Banker, G. (1998) Rat hippocampal neurons in low density culture. In Culturing Nerve Cells, G. Banker, and K. Goslin, eds., (Cambridge: MIT Press), pp. 339370

Hakomori, S. (2000).Traveling for the glycosphingolipid path. Glycoconj. J. 17, 627-647.

Hart, G.W., Slawson, C., Ramirez-Correa, G., and Lagerlof, O. (2011). Cross talk between O-GlcNAcylation and phosphorylation: roles in signaling, transcription, and chronic disease. Annu. Rev. Biochem. 80, 825-858.

Hinderlich, S. Nöhring, S., Weise, C., Franke, P., Stäsche, R , and Reutter, W. (1998). Purification and characterization of $N$-acetylglucosamine kinase from rat liver: comparison with UDP-Nacetylglucosamine 2-epimerase/ $\mathrm{N}$-acetylmannosamine kinase. Eur. J. Biochem. 252, 133-139.

Hinderlich, S., Berger, M., Schwarzkopf, M., Effertz, K., and Reutter, W. (2000). Molecular cloning and characterization of murine and human $N$-acetylglucosamine kinase. Eur. J. Biochem. 267, 3301-3308.

Hurley, J.H. (1996). The sugar kinase/heat shock protein 70/actin superfamily: implications of conserved structure for mechanism. Annu. Rev. Biophys. Biomol. Struct. 25, 137-162.

Jiang, M., Deng, L., and Chen, G. (2004). High Ca ${ }^{2+}$-phosphate transfection efficiency enables single neuron gene analysis.
Gene Ther. 11, 1303-1311.

Kreppel, L.K., and Hart, G.W. (1999). Regulation of a cytosolic and nuclear O-GIcNAc transferase. Role of the tetratricopeptide repeats. J. Biol. Chem. 274, 32015-32022

Ligos, J.M., de Lera, T.L., Hinderlich, S., Guinea, B., Sánchez, L., Roca, R., Valencia, A., and Bernad, A. (2002). Functional interaction between the Ser/Thr kinase PKL12 and N-acetylglucosamine kinase, a prominent enzyme implicated in the salvage pathway for GlcNAc recycling. J. Biol. Chem. 277, 6333-6343.

Moon, I.S., Cho, S.J., Jin, I., and Walikonis, R. (2007). A simple method for combined fluorescence in situ hybridization and immunocytochemistry. Mol. Cells 24, 76-82.

Moon, I.S., Lee, H.S., and Lee, H.J. (2013). The Potential 'OGlcNAc-P'om'. J. Life Sci. 23, 324-331.

O'Rourke, N.A., Cline, H.T., and Fraser, S.E. (1994). Rapid remodeling of retinal arbors in the tectum with and without blockade of synaptic transmission. Neuron 4, 921-934.

Schachter, H. (2000). The joys of HexNAc. The synthesis and function of $\mathrm{N}$ - and O-glycan branches. Glycoconj. J. 17, 465-483.

Sholl, D.A. (1953). Dendritic organization in the neurons of the visual and motor cortices of the cat. J. Anat. 87, 387-406.

Van den Steen, P., Rudd, P.M, Dwek, R.A., and Opdenakker, G. (1998). Concepts and principles of O-linked glycosylation. Crit. Rev. Biochem. Mol. Biol. 33, 151-208.

Watanabe, R., Murakami, Y., Marmor, M.D., Inoue, N., Maeda, Y., Hino, J., Kangawa, K., Julius, M., and Kinoshita, T. (2000). Initial enzyme for glycosylphosphatidylinositol biosynthesis requires PIG-P and is regulated by DPM2. EMBO J. 19, 4402-4411.

Weihofen, W.A., Berger, M., Chen, H., Saenger, W., and Hinderlich, $\mathrm{S}$. (2006). Structures of human $\mathrm{N}$-acetylglucosamine kinase in two complexes with $N$-acetylglucosamine and with ADP/glucose: insights into substrate specificity and regulation. J. Mol. Biol. 364, 388-399.

Yu, J.Y., DeRuiter, S.L., and Turner, D.L. (2002). RNA interference by expression of short-interfering RNAs and hairpin RNAs in mammalian cells. Proc. Natl. Acad. Sci. USA 99, 6047-6052. 